\title{
Attitude Determination and Control System for Satellites in Elliptical Orbits - a Complete Solution
}

\author{
Espen Oland \\ Department of Electrical Engineering \\ UiT - The Arctic University of Norway \\ Narvik, Norway \\ Email: espen.oland@uit.no
}

\begin{abstract}
This paper presents a complete solution to the problem of attitude determination and control for satellites in elliptical orbits. Specifically, it shows how to create the orbital mechanics, account for perturbing gravity torques, create a nonlinear PD+ attitude controller, map the control signal to desired thrust firings, implement magnetic field and Sun vector models, and how to implement a Madgwick filter for attitude determination based on vector measurements.
\end{abstract}

\section{INTRODUCTION}

The problem of developing Attitude Determination and Control Systems (ADCS) has received much attention the last century with general books such as [1], [2], [3] and [4], as well as description of individual ADCS designs for different satellites in works such as [5], [6], [7] and [8]. This paper builds on much of the previous work, as well as the research done through the HiNCube project as presented in [9], [10] and [11]. This work considers the problem of designing a complete ADCS system comprising of all the required components. Figure 1 shows the control structure and the required signal paths, giving an overview of the contents in this paper, as each block is described in detail to put the reader in position to design their own ADCS system.

\section{A. Notation}

This section is similar to [12]. The time derivative of a vector is denoted as $\dot{\mathbf{x}}=d \mathbf{x} / d t$ and the Euclidean length is written as $\|\mathbf{x}\|=\sqrt{\mathbf{x}^{\top} \mathbf{x}}$. Superscript denotes the reference frame of a vector. The rotation matrix is denoted $\mathbf{R}_{a}^{c} \in \mathcal{S O}(3)=\left\{\mathbf{R} \in \mathbb{R}^{3 \times 3}: \mathbf{R}^{\top} \mathbf{R}=\mathbf{I}, \operatorname{det}(\mathbf{R})=1\right\}$, which rotates a vector from frame $a$ to frame $c$ and where I denotes the identity matrix. The angular velocity vector is denoted $\boldsymbol{\omega}_{a, c}^{e}$, which represents the angular velocity of frame $c$ relative to frame $a$ referenced in frame $e$. Angular velocities between different frames can be added together as $\boldsymbol{\omega}_{a, f}^{e}=\boldsymbol{\omega}_{a, c}^{e}+\boldsymbol{\omega}_{c, f}^{e}$ (cf. [13]). The time derivative of the rotation matrix is found as $\dot{\mathbf{R}}_{a}^{c}=\mathbf{R}_{a}^{c} \mathbf{S}\left(\boldsymbol{\omega}_{c, a}^{a}\right)$ where the cross product operator $\mathbf{S}(\cdot)$ is such that for two vectors $\mathbf{v}_{1}, \mathbf{v}_{2} \in \mathbb{R}^{3}, \mathbf{S}\left(\mathbf{v}_{1}\right) \mathbf{v}_{2}=\mathbf{v}_{1} \times \mathbf{v}_{2}, \mathbf{S}\left(\mathbf{v}_{1}\right) \mathbf{v}_{2}=-\mathbf{S}\left(\mathbf{v}_{2}\right) \mathbf{v}_{1}$, $\mathbf{S}\left(\mathbf{v}_{1}\right) \mathbf{v}_{1}=\mathbf{0}$ and $\mathbf{v}_{1}^{\top} \mathbf{S}\left(\mathbf{v}_{2}\right) \mathbf{v}_{1}=0$. The rotation matrix can be parameterized using quaternions ( $c f$. [14]), where the quaternion that represents a rotation from frame $a$ to frame $c$ is denoted $\mathbf{q}_{c, a} \in \mathcal{S}^{3}=\left\{\mathbf{q} \in \mathbb{R}^{4}: \mathbf{q}^{\top} \mathbf{q}=1\right\}$ and can be written as $\mathbf{q}_{c, a}=\left[\begin{array}{ll}\eta_{c, a} & \boldsymbol{\epsilon}_{c, a}^{\top}\end{array}\right]^{\top}=\left[\begin{array}{ll}\cos \left(\frac{\vartheta_{c, a}}{2}\right) & \mathbf{k}_{c, a}^{\top} \sin \left(\frac{\vartheta_{c, a}}{2}\right)\end{array}\right]^{\top}$ which performs a rotation of an angle $\vartheta_{c, a}$ around the unit vector $\mathbf{k}_{c, a}$, and the inverse quaternion is defined as $\mathbf{q}_{a, c}=\left[\begin{array}{ll}\eta_{c, a} & -\boldsymbol{\epsilon}_{c, a}^{\top}\end{array}\right]^{\top}, \mathbf{q}^{*}$ is also used to denote the inverse quaternion. The scalar part is denoted $\eta_{c, a}$ and the vector part as $\boldsymbol{\epsilon}_{c, a} \in \mathbb{R}^{3}$, enabling the rotation matrix to be constructed as $\mathbf{R}_{a}^{c}=\mathbf{I}+2 \eta_{c, a} \mathbf{S}\left(\boldsymbol{\epsilon}_{c, a}\right)+2 \mathbf{S}^{2}\left(\boldsymbol{\epsilon}_{c, a}\right)$. Composite rotations are found using the quaternion product as (cf. [13]) $\mathbf{q}_{c, e}=\mathbf{q}_{c, a} \otimes \mathbf{q}_{a, e}=\mathbf{T}\left(\mathbf{q}_{c, a}\right) \mathbf{q}_{a, e}$ with

$$
\mathbf{T}\left(\mathbf{q}_{c, a}\right)=\left[\begin{array}{cc}
\eta_{c, a} & -\boldsymbol{\epsilon}_{c, a}^{\top} \\
\boldsymbol{\epsilon}_{c, a} & \eta_{c, a} \mathbf{I}+\mathbf{S}\left(\boldsymbol{\epsilon}_{c, a}\right)
\end{array}\right],
$$

which ensures that the resulting quaternion maintains the unit length property, while the quaternion kinematics is given as

$$
\dot{\mathbf{q}}_{c, a}=\frac{1}{2} \mathbf{q}_{c, a} \otimes\left[\begin{array}{c}
0 \\
\boldsymbol{\omega}_{c, a}^{a}
\end{array}\right]=\frac{1}{2} \mathbf{T}\left(\mathbf{q}_{c, a}\right)\left[\begin{array}{c}
0 \\
\boldsymbol{\omega}_{c, a}^{a}
\end{array}\right] .
$$

\section{B. Reference Frames}

Inertial: The Earth Centered Inertial (ECI) is denoted by $\mathcal{F}^{i}$ and has its origin in the center of the Earth. The $\mathrm{x}^{i}$ axis points towards the Vernal equinox, $\mathbf{z}^{i}$ points through the north pole, and $\mathbf{y}^{i}$ completes the right-handed orthonormal frame.

Orbit: The orbit frame is denoted by $\mathcal{F}^{o}$ and has the origin in the center of mass of the satellite ( $c f .[15$, p. 479]). The $\mathbf{e}_{r}$ axis coincides with the radius vector $\mathbf{r}^{i} \in \mathbb{R}^{3}$ going from the center of the Earth to the center of mass in the satellite. The $\mathbf{e}_{h}$ axis is parallel to the orbital angular momentum vector, pointing in the normal direction of the orbit. The $\mathbf{e}_{\theta}$ completes the right-handed orthonormal frame and the vectors can be described as: $\mathbf{e}_{r}=\frac{\mathbf{r}^{i}}{\left\|\mathbf{r}^{i}\right\|}, \mathbf{e}_{\theta}=\mathbf{e}_{h} \times \mathbf{e}_{r}$ and $\mathbf{e}_{h}=\frac{\mathbf{h}}{\|\mathbf{h}\|}$ where $\mathbf{h}=\mathbf{r}^{i} \times \dot{\mathbf{r}}^{i}$.

Body: The body frame is denoted by $\mathcal{F}^{b}$ and has its origin in center of mass of the satellite, where its axes coincide with the principal axes of inertia of the satellite.

Desired: The desired frame is denoted by $\mathcal{F}^{d}$ and can be defined arbitrarily. In this work it is simply set to a constant orientation, but it can be defined to achieve multiple objectives as described in [16].

\section{ORbital Mechanics}

This section relates the orbit frame to the inertial frame and presents how to find the radius, velocity, acceleration, angular velocity and angular acceleration vectors to properly describe the satellite's location in the orbit. The six classical 


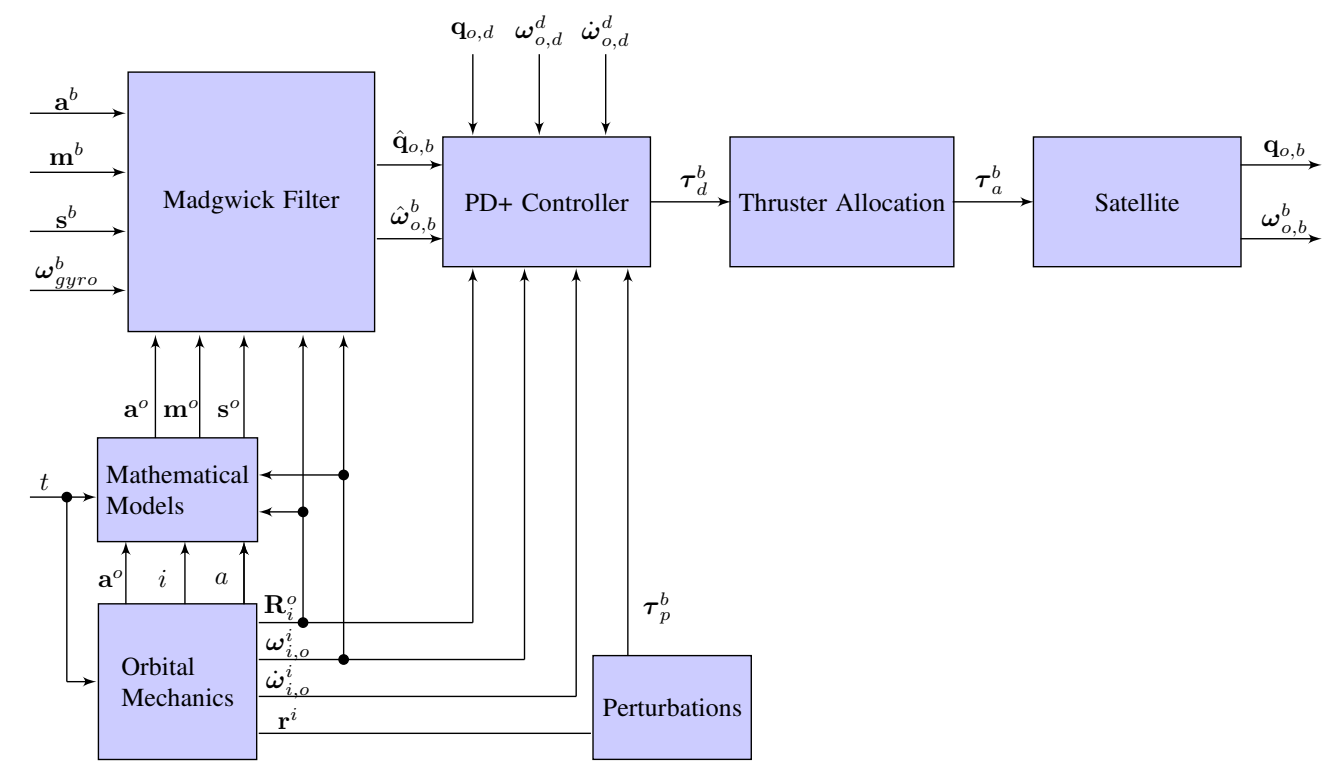

Fig. 1. Control structure. The required measurements are the magnetic field, the acceleration vector, the angular velocity, the Sun vector and the current time; where the current time serve as input to the mathematical models of the Sun vector, magnetic field and acceleration vector, which are required for attitude determination. By using the information about perturbing torques, orbital mechanics and current attitude and angular velocity, the control signal can be calculated using a PD+ controller, and mapped to thruster firings. This allows the satellite to obtain a desired attitude and angular velocity.

orbit parameters are defined as: the semimajor axis $a$, the eccentricity $e$, the inclination $i$, the right ascension of the ascending node $\Omega$, the argument of the perigee $\omega$, and the mean anomaly $M$. Given the radius from the center of the Earth to the perigee and apogee defined by $r_{p}$ and $r_{a}$ respectively, then the semimajor axis can be found as $a=\frac{r_{a}+r_{p}}{2}$, the eccentricity as $e=\frac{r_{a}-r_{p}}{r_{a}+r_{p}}$ and the mean motion as $n=\sqrt{\frac{\mu}{a^{3}}}$ where $\mu=G M_{\text {Earth }}$ with $G$ as the gravitational constant and $M_{\text {Earth }}$ as the mass of the Earth. From this, the mean anomaly can be found as $M=n\left(t-t_{0}\right)=\psi-e \sin (\psi)$ where $t$ is the time and $t_{0}$ is the time of passage of the perigee, and $\psi$ is the eccentric anomaly. Further, to find where in the orbit the satellite is located, the true anomaly $\theta$ is required, which can be found as $\theta=\cos ^{-1}\left(\frac{\cos (\psi)-e}{1-e \cos (\psi)}\right)$, which can be differentiated to find the rate as $[17, \mathrm{p} .42] \dot{\theta}=\frac{n(1+e \cos (\theta))^{2}}{\left(1-e^{2}\right)^{\frac{3}{2}}}$, which allows for easy integration when calculating the orbital position. It is however, not possible to solve the eccentric anomaly analytically, such that it must be solved using an iterative algorithm as [1, p.26] $\psi_{k}(t)=M(t)+e \sin \left(\psi_{k-1}(t)\right)$, where $k$ is the iteration number and the algorithm is initialized with $\psi_{0}\left(t_{0}\right)=M\left(t_{0}\right)$, and allowed to run until the difference $\left|\psi_{k}(t)-\psi_{k-1}(t)\right| \leq 1 \cdot 10^{-6}$. Note that this algorithm is valid as long as $0<e<1$, which holds for elliptical orbits. The six classical orbit parameters together with the true anomaly now allow the rotation matrix from the inertial to the orbit frame to be constructed as given in equation (8). Further, the radius vector to the satellite in the orbit frame is found as $[1, \mathrm{p} .26]$

$$
\mathbf{r}^{o}=\left[\begin{array}{lll}
a \cos (\psi)-a e & a \sin (\psi) \sqrt{1-e^{2}} & 0
\end{array}\right]^{\top}
$$

where it can be rotated to the inertial frame as $\mathbf{r}^{i}=\mathbf{R}_{i}^{o} \mathbf{r}^{o}$, and has length $r=\left\|\mathbf{r}^{i}\right\|$. The velocity vector in the orbit frame can be found as [1, p.27]

$$
\mathbf{v}^{o}=\left[\begin{array}{lll}
-\frac{a^{2} n}{r} \sin (\psi) & \frac{a^{2} n}{r} \sqrt{1-e^{2}} \cos (\psi) & 0
\end{array}\right]^{\top}
$$

which can be rotated to the inertial frame as $\mathbf{v}^{i}=\mathbf{R}_{o}^{i} \mathbf{v}^{o}$, and finally the acceleration vector can be obtained through differentiation as

$$
\mathbf{a}^{o}=\left[\begin{array}{lll}
-\frac{a^{3} n^{2}}{r^{2}} \cos (\psi) & -\frac{a^{3} n^{2}}{r^{2}} \sqrt{1-e^{2}} \sin (\psi) \quad 0
\end{array}\right]^{\top}
$$

and in the inertial frame as $\mathbf{a}^{i}=\mathbf{R}_{o}^{i} \mathbf{a}^{o}$. From (9)-(11), the angular velocity of the orbit frame relative to the inertial frame can be found as $\boldsymbol{\omega}_{i, o}^{i}=\frac{\mathbf{r}^{i} \times \mathbf{v}^{i}}{\left(\mathbf{r}^{i}\right)^{\top} \mathbf{r}^{i}}$ and the angular acceleration as $\dot{\boldsymbol{\omega}}_{i, o}^{i}=\frac{\left(\mathbf{r}^{i} \times \mathbf{a}^{i}\right)\left(\mathbf{r}^{i}\right)^{\top} \mathbf{r}^{i}-2\left(\mathbf{r}^{i} \times \mathbf{v}^{i}\right)\left(\mathbf{v}^{i}\right)^{\top} \mathbf{r}^{i}}{\left(\left(\mathbf{r}^{i}\right)^{\top} \mathbf{r}^{i}\right)^{2}}$.

\section{Sun VECTOR MODEL}

To find the direction towards the Sun, it is possible to apply the Sun vector model presented in [3, pp.281 - 282], which is valid until the year 2050 and has an accuracy of $0.01^{\circ}$. First the current time and date must be converted into the

$$
\mathbf{R}_{i}^{o}=\left[\begin{array}{ccc}
\cos (\omega+\theta) \cos (\Omega)-\cos (i) \sin (\omega+\theta) \sin (\Omega) & \cos (\omega+\theta) \sin (\Omega)+\sin (\omega+\theta) \cos (i) \cos (\Omega) & \sin (\omega+\theta) \sin (i) \\
-\sin (\omega+\theta) \cos (\Omega)-\cos (i) \sin (\Omega) \cos (\omega+\theta) & -\sin (\omega+\theta) \sin (\Omega)+\cos (\omega+\theta) \cos (i) \cos (\Omega) & \cos (\omega+\theta) \sin (i) \\
\sin (i) \sin (\Omega) & -\sin (i) \cos (\Omega) & \cos (i)
\end{array}\right]
$$


Julian date through the algorithm (12) ( $c f$. [3, p.189]). Here $I N T()$ denotes a real truncation; $y r, m o, d, h, \min , s$ denote the year, month, day, hour, minute and second, respectively, and if the day contains a leap second, use 61 instead of $60 *$. From the Julian Date $(J D)$, the Sun vector model can now be constructed after doing some simplifications as:

$$
\begin{aligned}
& T_{U T 1}=\frac{J D-2,451,545.0}{36,525} \\
& \lambda_{M_{\odot}}=280.460^{\circ}+36,000.771 T_{U T 1} \\
& M_{\odot}=357.5277233^{\circ}+35,999.05034 T_{U T 1} \\
& \lambda_{\text {ecliptic }}=\lambda_{M_{\odot}}+1.914666471^{\circ} \sin \left(M_{\odot}\right) \\
& +0.019994643 \sin \left(2 M_{\odot}\right) \\
& \epsilon=23.439291^{\circ}-0.0130042 T_{U T 1} \\
& \mathbf{s}^{o}=\mathbf{R}_{i}^{o}\left[\begin{array}{c}
\cos \left(\lambda_{\text {ecliptic }}\right) \\
\cos (\epsilon) \sin \left(\lambda_{\text {ecliptic }}\right) \\
\sin (\epsilon) \sin \left(\lambda_{\text {ecliptic }}\right)
\end{array}\right]
\end{aligned}
$$

where $T_{U T 1}$ is the number of Julian centuries, $\lambda_{M_{\odot}}$ is the mean longitude of the Sun, $M_{\odot}$ is the mean anomaly for the Sun, $\lambda_{\text {ecliptic }}$ is the ecliptic longitude, $\epsilon$ is the obliquity of the ecliptic, and $\mathbf{s}^{o}$ is the Sun vector in orbit frame. Note that the angles are defined in degrees, and must be converted to radians before using the trigonometric functions. Hence, a relation between the Julian Date and the direction towards the Sun has been established, and will be used for attitude determination.

\section{Magnetic Field Model}

There are many different geomagnetic models that can be applied for attitude determination, ranging from advanced models such as the 12th generation IGRF model [18] to simpler dipole models [19]. In this section a simple dipole model will be presented and used for attitude determination, where the underlying assumption is no Earth rotation and no orbit precession. This gives the magnetic field as [19]

$$
\mathbf{m}^{o}=\frac{\mu_{f}}{a^{3}}\left[\cos \left(\omega_{0} t\right) \sin (i) \quad-\cos (i) \quad 2 \sin \left(\omega_{0} t\right) \sin (i)\right]^{\top}
$$

where $t$ is the time measured from passing the ascending node of the orbit relative to the geomagnetic equator, $\mu_{f}$ is the dipole strength, and the orbit's angular speed is $\omega_{0}=\left\|\boldsymbol{\omega}_{i, o}^{i}\right\|$.

\section{AtTitude Dynamics ANd CONTROL}

The attitude dynamics can be written relative to the inertial frame using Euler's moment equation as

$$
\mathbf{J} \dot{\boldsymbol{\omega}}_{i, b}^{b}=-\mathbf{S}\left(\boldsymbol{\omega}_{i, b}^{b}\right) \mathbf{J} \boldsymbol{\omega}_{i, b}^{b}+\boldsymbol{\tau}_{a}^{b}+\boldsymbol{\tau}_{p}^{b}
$$

where $\tau_{a}^{b} \in \mathbb{R}^{3}$ denotes the actuation torques (e.g. output from reaction wheels), while $\tau_{p}^{b} \in \mathbb{R}^{3}$ denotes the perturbing torques (e.g. gravity torque). For attitude control, it might be more interesting controlling the attitude relative to the orbit frame (e.g. to enable nadir pointing), and the angular velocity of the body frame relative to the orbit frame can be found as

$$
\boldsymbol{\omega}_{o, b}^{b}=\boldsymbol{\omega}_{i, b}^{b}-\mathbf{R}_{i}^{b} \boldsymbol{\omega}_{i, o}^{i}
$$

and can be differentiated as

$$
\begin{aligned}
\mathbf{J} \dot{\boldsymbol{\omega}}_{o, b}^{b}= & -\mathbf{S}\left(\boldsymbol{\omega}_{i, b}^{b}\right) \mathbf{J} \boldsymbol{\omega}_{i, b}^{b}+\boldsymbol{\tau}_{a}^{b}+\boldsymbol{\tau}_{p}^{b}+\mathbf{J S}\left(\boldsymbol{\omega}_{i, b}^{b}\right) \mathbf{R}_{i}^{b} \boldsymbol{\omega}_{o, i}^{i} \\
& -\mathbf{J} \mathbf{R}_{i}^{b} \dot{\boldsymbol{\omega}}_{i, o}^{i} .
\end{aligned}
$$

Further, to enable tracking of a desired quaternion and angular velocity defined relative to the orbit frame, let $\mathbf{q}_{o, d}, \boldsymbol{\omega}_{o, d}^{d}, \dot{\boldsymbol{\omega}}_{o, d}^{d} \in \mathcal{L}_{\infty}$ denote a desired trajectory, then the quaternion and angular velocity error can be found as

$$
\begin{aligned}
\mathbf{q}_{d, b} & =\mathbf{q}_{d, o} \otimes \mathbf{q}_{o, b} \\
\boldsymbol{\omega}_{d, b}^{b} & =\boldsymbol{\omega}_{o, b}^{b}-\mathbf{R}_{d}^{b} \boldsymbol{\omega}_{o, d}^{d},
\end{aligned}
$$

which holds the kinematics as ( $c f$. equation (2))

$$
\begin{aligned}
& \dot{\eta}_{d, b}=-\frac{1}{2} \boldsymbol{\epsilon}_{d, b}^{\top} \boldsymbol{\omega}_{d, b}^{b} \\
& \dot{\boldsymbol{\epsilon}}_{d, b}=\left(\eta_{d, b} \mathbf{I}+\mathbf{S}\left(\boldsymbol{\epsilon}_{d, b}\right)\right) \boldsymbol{\omega}_{d, b}^{b},
\end{aligned}
$$

while the angular acceleration error can be found as

$$
\begin{aligned}
\mathbf{J} \dot{\boldsymbol{\omega}}_{d, b}^{b}= & -\mathbf{S}\left(\boldsymbol{\omega}_{i, b}^{b}\right) \mathbf{J} \boldsymbol{\omega}_{i, b}^{b}+\boldsymbol{\tau}_{a}^{b}+\boldsymbol{\tau}_{p}^{b}+\mathbf{J S}\left(\boldsymbol{\omega}_{i, b}^{b}\right) \mathbf{R}_{i}^{b} \boldsymbol{\omega}_{o, i}^{i} \\
& -\mathbf{J} \mathbf{R}_{i}^{b} \dot{\boldsymbol{\omega}}_{i, o}^{i}+\mathbf{J S}\left(\boldsymbol{\omega}_{o, b}^{b}\right) \mathbf{R}_{d}^{b} \boldsymbol{\omega}_{o, d}^{d}-\mathbf{J} \mathbf{R}_{d}^{b} \dot{\boldsymbol{\omega}}_{o, d}^{d}
\end{aligned}
$$

\section{A. PD+ Attitude Controller}

Paden and Panja presented in [20] a passivity-based approach for controlling robotic manipulators. This has later been applied for spacecraft in [21] showing very good performance. A similar controller will be derived in this section, also sharing some similarities with [12]. Let a Lyapunov Function Candidate be chosen as $V=\frac{1}{2}\left(\boldsymbol{\omega}_{d, b}^{b}\right)^{\top} \mathbf{J} \boldsymbol{\omega}_{d, b}^{b}+k_{p}\left(1-\eta_{d, b}\right)^{2}+$ $k_{p} \boldsymbol{\epsilon}_{d, b}^{\top} \boldsymbol{\epsilon}_{d, b}$ where $k_{p}$ is a positive scalar gain. Its derivative is found using (25)-(27) as

$$
\begin{aligned}
\dot{V}= & k_{p} \boldsymbol{\epsilon}_{d, b}^{\top} \boldsymbol{\omega}_{d, b}^{b}+\left(\boldsymbol{\omega}_{d, b}^{b}\right)^{\top}\left(-\mathbf{S}\left(\boldsymbol{\omega}_{i, b}^{b}\right) \mathbf{J} \boldsymbol{\omega}_{i, b}^{b}+\boldsymbol{\tau}_{a}^{b}+\boldsymbol{\tau}_{p}^{b}\right. \\
& +\mathbf{J S}\left(\boldsymbol{\omega}_{i, b}^{b}\right) \mathbf{R}_{i}^{b} \boldsymbol{\omega}_{o, i}^{i}-\mathbf{J} \mathbf{R}_{i}^{b} \dot{\boldsymbol{\omega}}_{i, o}^{i} \\
& \left.+\mathbf{J S}\left(\boldsymbol{\omega}_{o, b}^{b}\right) \mathbf{R}_{d}^{b} \boldsymbol{\omega}_{o, d}^{d}-\mathbf{J R}_{d}^{b} \dot{\boldsymbol{\omega}}_{o, d}^{d}\right)
\end{aligned}
$$

A PD+ control law can now be chosen as

$$
\begin{aligned}
\boldsymbol{\tau}_{d}^{b}= & \mathbf{J} \mathbf{R}_{d}^{b} \dot{\boldsymbol{\omega}}_{o, d}^{d}-\mathbf{J S}\left(\boldsymbol{\omega}_{o, b}^{b}\right) \mathbf{R}_{d}^{b} \boldsymbol{\omega}_{o, d}^{d}+\mathbf{J} \mathbf{R}_{i}^{b} \dot{\boldsymbol{\omega}}_{i, o}^{i} \\
& -\mathbf{J S}\left(\boldsymbol{\omega}_{i, b}^{b}\right) \mathbf{R}_{i}^{b} \boldsymbol{\omega}_{o, i}^{i}-\boldsymbol{\tau}_{p}^{b}+\mathbf{S}\left(\boldsymbol{\omega}_{i, b}^{b}\right) \mathbf{J} \boldsymbol{\omega}_{i, b}^{b} \\
& -k_{p} \boldsymbol{\epsilon}_{d, b}-k_{d} \boldsymbol{\omega}_{d, b}^{b}
\end{aligned}
$$

where $k_{d}$ is another positive scalar gain, and $\tau_{d}^{b}$ denotes the desired torque required to achieve the objective. Assuming

$$
J D=367(y r)-I N T\left(\frac{7\left(y r+I N T\left(\frac{m o+9}{12}\right)\right)}{4}\right)+I N T\left(\frac{275 m o}{9}\right)+d+1,721,013.5+\frac{\frac{\left(\frac{s}{60 *}+m i n\right)}{60}+h}{24}
$$


no actuator dynamics, i.e. $\boldsymbol{\tau}_{a}^{b}=\boldsymbol{\tau}_{d}^{b}$, then by inserting (29) into (28), it is obtained that $\dot{V} \leq-k_{d}\left\|\boldsymbol{\omega}_{d, b}^{b}\right\|^{2}$, which is negative semi-definite. By applying the Matrosov theorem (cf. [22]) it can be shown that the origin $\left(\boldsymbol{\epsilon}_{d, b}, \boldsymbol{\omega}_{d, b}^{b}\right)=(\mathbf{0}, \mathbf{0})$ is uniformly asymptotically stable, such that the attitude and angular velocity will track the desired values asymptotically.

\section{B. Actuators}

There are different kinds of actuators that can be used for attitude control of a satellite, such as thrusters, magnetic torquers or reaction wheels. This section presents how to map the control signal (29) to four thrusters used for attitude control. Let each thruster have a location relative to the center of mass denoted by $\mathbf{r}_{i}^{b}=\left[\begin{array}{lll}r_{x} & r_{y} & r_{z}\end{array}\right]^{\top}$ and with an azimuth and an elevation angle described by $\gamma$ and $\chi$. Then the torque produced by the i'th thruster can be described as [1, p. 262]

$$
\boldsymbol{\tau}_{i}^{b}=\mathbf{r}_{i}^{b} \times \mathbf{f}_{i}^{b}=\left[\begin{array}{c}
r_{y} \sin (\gamma) \cos (\chi)-r_{z} \sin (\chi) \\
r_{z} \cos (\chi) \cos (\gamma)-r_{x} \cos (\chi) \sin (\gamma) \\
r_{x} \sin (\chi)-r_{y} \cos (\chi) \cos (\gamma)
\end{array}\right] f_{i}
$$

where $f_{i}$ denotes the total thrust from the i'th thruster. Now, let the the thruster configuration be defined as given in Table I, then the torque can be written as $\boldsymbol{\tau}_{a}^{b}=\mathbf{B u}$ where the control allocation matrix can be constructed as

$$
\mathbf{B}=\left[\begin{array}{cccc}
-\frac{\sqrt{2}}{\sqrt{5}} & \frac{\sqrt{2}}{5} & \frac{\sqrt{2}}{5} & -\frac{\sqrt{2}}{5} \\
\frac{\sqrt{2}}{4} & -\frac{\sqrt{2}}{4} & \frac{\sqrt{2}}{4} & -\frac{\sqrt{2}}{4} \\
-\frac{\sqrt{2}}{4} & -\frac{\sqrt{2}}{4} & \frac{\sqrt{2}}{4} & \frac{\sqrt{2}}{4}
\end{array}\right]
$$

and the vector of thruster signals as $\mathbf{u}=\left[\begin{array}{llll}f_{1} & f_{2} & f_{3} & f_{4}\end{array}\right]^{\top}$. After finding the control signal (29), it can be mapped to find the desired thrust values as $\mathbf{u}_{d}=\mathbf{B}^{\dagger} \boldsymbol{\tau}_{d}^{b}$, where $\dagger$ denotes the Moore-Penrose pseudoinverse and $\mathbf{u}_{d}=$ $\left[\begin{array}{llll}u_{1} & u_{2} & u_{3} & u_{4}\end{array}\right]^{\top}$. Then the $\mathbf{u}$ vector can be modeled using Bang-bang modulation, i.e. the thruster if fully actuated as long as the i'th signal of $\mathbf{u}_{d}$ is above zero, such that

$$
f_{i}= \begin{cases}f_{\max } & \text { if } u_{i}>0 \\ 0 & \text { if } u_{i} \leq 0\end{cases}
$$

where $f_{\max }$ denotes the maximum available thrust from the i'th thruster. After applying bang-bang modulation, the vector $\mathbf{u}$ can be constructed allowing the actuator torque to be found as $\tau_{a}^{b}=\mathbf{B u}$. Alternatives to bang-bang modulation are Pulse Width Modulation (PWM) and Pulse Width Pulse Frequency Modulation (PWPF), which are known to provide better performance.
TABLE I

THRUSTER CONFIGURATION

\begin{tabular}{c|c|c|r|r|r} 
Thruster & Elevation $(\chi)$ & Azimuth $(\gamma)$ & $r_{x}$ & $r_{y}$ & $r_{z}$ \\
\hline$f_{1}$ & 45 & 90 & -0.5 & -0.45 & -0.05 \\
$f_{2}$ & 135 & 90 & -0.5 & -0.45 & 0.05 \\
$f_{3}$ & -45 & 90 & -0.5 & 0.45 & -0.05 \\
$f_{4}$ & -135 & 90 & -0.5 & 0.45 & 0.05 \\
\hline
\end{tabular}

\section{Perturbing Torques}

There are different kinds of perturbing torques, such as gravity torque, aerodynamic torque, magnetic field due to the electronics inside the satellite, as well as solar radiation torque. This section only considers the gravity torque, which is given as [15, p. 147] $\boldsymbol{\tau}_{g}^{b}=\frac{3 G M_{\text {Earth }}}{r^{5}} \mathbf{r}^{i} \times \mathbf{J} \mathbf{r}^{i}$ where $G$ is the gravitational constant, $M_{\text {Earth }}$ is the mass of the Earth and $\mathbf{J}$ is the inertia of the satellite, indicating that for nondiagonal inertia matrices, the gravity will induce torques to align the satellite with the gravity field. Hence, for this paper the perturbing torque is given by $\tau_{p}^{b}=\tau_{g}^{b}$.

\section{AtTitude Determination}

The Madgwick filter presented in [23], shows good results in estimating the orientation based on IMU measurement. It is based on using gradient descent in combination with complementary filter to fuse sensor data together to produce the estimated quaternion. Alternatives are the TRIAD method [24], by using the Kalman filter [25] or the Mahony filter [26]. In this section, the Madgwick filter is adapted to the case of attitude determination for satellites by including information about the Sun vector. Let $\hat{\mathbf{q}}=\left[\begin{array}{llll}q_{1} & q_{2} & q_{3} & q_{4}\end{array}\right]^{\top}$ denote the quaternion estimate, $\mathbf{a}^{b}, \mathbf{s}^{b}$ and $\mathbf{m}^{b}$ are the measured acceleration, Sun vector and magnetic field respectively, and can be combined with the mathematical models of the same vectors in the orbit frame given in (11), (18) and (19). Subscript $k$ denotes the current estimate, while $k-1$ denotes the previous estimate. First an objective function can be defined as

$$
\mathcal{F}=\left[\begin{array}{c}
\mathbf{f}\left(\hat{\mathbf{q}}_{k-1}, \mathbf{a}^{o}, \mathbf{a}^{b}\right) \\
\mathbf{f}\left(\hat{\mathbf{q}}_{k-1}, \mathbf{s}^{o}, \mathbf{s}^{b}\right) \\
\mathbf{f}\left(\hat{\mathbf{q}}_{k-1}, \mathbf{m}^{o}, \mathbf{m}^{b}\right)
\end{array}\right]
$$

where the objective is find an estimated quaternion that minimizes this function, something that can be achieved by using gradient descent. The Jacobian matrix can be obtained as

$$
\mathcal{J}=\left[\begin{array}{c}
\mathbf{J}_{q}\left(\hat{\mathbf{q}}_{k-1}, \mathbf{a}^{o}\right) \\
\mathbf{J}_{q}\left(\hat{\mathbf{q}}_{k-1}, \mathbf{s}^{o}\right) \\
\mathbf{J}_{q}\left(\hat{\mathbf{q}}_{k-1}, \mathbf{m}^{o}\right)
\end{array}\right]
$$

$$
\begin{gathered}
\mathbf{f}\left(\hat{\mathbf{q}}_{k-1}, \mathbf{z}^{o}, \mathbf{z}^{b}\right)=\left[\begin{array}{lll}
2 o_{x}\left(0.5-q_{3}^{2}-q_{4}^{2}\right)+2 o_{y}\left(q_{1} q_{4}+q_{2} q_{3}\right)+2 o_{z}\left(q_{2} q_{4}-q_{1} q_{3}\right)-b_{x} \\
2 o_{x}\left(q_{2} q_{3}-q_{1} q_{4}\right)+2 o_{y}\left(0.5-q_{2}^{2}-q_{4}^{2}\right)+2 o_{z}\left(q_{1} q_{2}+q_{3} q_{4}\right)-b_{y} \\
2 o_{x}\left(q_{1} q_{3}+q_{2} q_{4}\right)+2 o_{y}\left(q_{3} q_{4}-q_{1} q_{2}\right)+2 o_{z}\left(0.5-q_{2}^{2}-q_{3}^{2}\right)-b_{z}
\end{array}\right] \\
\mathbf{J}_{q}\left(\hat{\mathbf{q}}_{k-1}, \mathbf{z}^{o}\right)=\left[\begin{array}{cccc}
2 o_{y} q_{4}-2 o_{z} q_{3} & 2 o_{y} q_{3}+2 o_{z} q_{4} & -4 o_{x} q_{3}+2 o_{y} q_{2}-2 o_{z} q_{1} & -4 o_{x} q_{4}+2 o_{y} q_{1}+2 o_{z} q_{2} \\
-2 o_{x} q_{4}+2 o_{z} q_{2} & 2 o_{x} q_{3}-4 o_{y} q_{2}+2 o_{z} q_{1} & 2 o_{x} q_{2}+2 o_{z} q_{4} & -2 o_{x} q_{1}-4 o_{y} q_{4}+2 o_{z} q_{3} \\
2 o_{x} q_{3}-2 o_{y} q_{2} & 2 o_{x} q_{4}-2 o_{y} q_{1}-4 o_{z} q_{2} & 2 o_{x} q_{1}+2 o_{y} q_{4}-4 o_{z} q_{3} & 2 o_{x} q_{2}+2 o_{y} q_{3}
\end{array}\right]
\end{gathered}
$$


and allows the gradient to be found as $\nabla \mathbf{f}=\mathcal{J}^{\top} \mathcal{F}$. Now, let $\mathbf{z}^{o}=\left[\begin{array}{lll}o_{x} & o_{y} & o_{z}\end{array}\right]^{\top}$ denote a vector in the orbit frame obtained from a model, and $\mathbf{z}^{b}=\left[\begin{array}{lll}b_{x} & b_{y} & b_{z}\end{array}\right]^{\top}$ denote a vector in the body frame obtained from sensor measurements. Then the functions $\mathbf{f}\left(\hat{\mathbf{q}}_{k-1}, \mathbf{z}^{o}, \mathbf{z}^{b}\right)$ and $\mathbf{J}_{q}\left(\hat{\mathbf{q}}_{k-1}, \mathbf{z}^{o}\right)$ are given by (35) and (36) on the previous page. The gyro measures the angular velocity of the body relative to the inertial frame. To relate the gyro measurement $\boldsymbol{\omega}_{\text {gyro }}^{b}$ to the orbit frame, let ( $c f$. (21))

$$
\boldsymbol{\omega}_{o, \text { gyro }}^{b}=\left[\begin{array}{c}
0 \\
\boldsymbol{\omega}_{\text {gyro }}^{b}-\mathbf{R}_{o}^{b}\left(\hat{\mathbf{q}}_{k-1}\right) \mathbf{R}_{i}^{o} \boldsymbol{\omega}_{i, o}^{i}
\end{array}\right] \in \mathbb{R}^{4}
$$

where $\mathbf{R}_{o}^{b}\left(\hat{\mathbf{q}}_{k-1}\right)$ is the rotation matrix from the orbit frame to the body frame using the estimated quaternion. The main filter can now be presented as

$$
\begin{aligned}
\hat{\boldsymbol{\omega}}_{k}^{b} & =2 \mathbf{T}\left(\hat{\mathbf{q}}_{k-1}^{*}\right) \frac{\nabla \mathbf{f}}{\|\nabla \mathbf{f}\|} \\
\boldsymbol{\omega}_{b i a s, k}^{b} & =\boldsymbol{\omega}_{b i a s, k-1}^{b}+\zeta \hat{\boldsymbol{\omega}}_{k}^{b} \Delta T \\
\boldsymbol{\omega}_{o, b}^{b} & =\mathbf{H}\left(\boldsymbol{\omega}_{o, g y r o}^{b}-\boldsymbol{\omega}_{b i a s, k}^{b}\right) \\
\dot{\hat{\mathbf{q}}}_{k} & =\frac{1}{2} \mathbf{T}\left(\hat{\mathbf{q}}_{k-1}\right)\left[\begin{array}{c}
0 \\
\boldsymbol{\omega}_{o, b}^{b}
\end{array}\right]-\beta \frac{\nabla \mathbf{f}}{\|\nabla \mathbf{f}\|} \\
\hat{\mathbf{q}}_{k} & =\hat{\mathbf{q}}_{k-1}+\dot{\hat{\mathbf{q}}}_{k} \Delta T \\
\hat{\mathbf{q}}_{k} & =\frac{\hat{\mathbf{q}}_{k}}{\left\|\hat{\mathbf{q}}_{k}\right\|}
\end{aligned}
$$

where $\beta$ and $\zeta$ are gains, $\Delta T$ denotes the time-step, $\hat{\boldsymbol{\omega}}_{k}^{b} \in$ $\mathbb{R}^{4}$ denotes an estimate of the angular velocity based on the measured vectors, $\boldsymbol{\omega}_{\text {bias }, k}^{b} \in \mathbb{R}^{4}$ is an estimate of the gyro bias, $\boldsymbol{\omega}_{o, b}^{b} \in \mathbb{R}^{3}$ is the angular velocity of the body relative to the orbit frame (expected output) and $\hat{\mathbf{q}}_{k}$ denotes the estimated quaternion of the body frame relative to the orbit frame. Since this filter uses the complementary filter approach in fusing the sensor measurements, the quaternion must be normalized to ensure unit length. Also note that $\hat{\boldsymbol{\omega}}_{k}^{b}, \boldsymbol{\omega}_{\text {bias }, k}^{b} \in \mathbb{R}^{4}$, such that their first element must be enforced to zero. The projection matrix $\mathbf{H}=\left[\begin{array}{ll}\mathbf{0} & \mathbf{I}\end{array}\right] \in \mathbb{R}^{3 \times 4}$ comprises of a column-vector of zeros followed by the identity matrix, allowing $\boldsymbol{\omega}_{o, b}^{b} \in \mathbb{R}^{3}$.

\section{Simulation}

Let a satellite have the inertia matrix

$$
\mathbf{J}=\left[\begin{array}{ccc}
0.5 & -0.2 & -0.1 \\
-0.2 & 0.5 & -0.2 \\
-0.1 & -0.2 & 0.5
\end{array}\right]
$$

which contains non-diagonal terms which therefore will create perturbing moments due to the gravity. Furthermore, let the initial conditions be $\mathbf{q}_{o, b}(0)=\left[\begin{array}{llll}0.5 & 0.5 & 0.5 & 0.5\end{array}\right]^{\top}$ and $\boldsymbol{\omega}_{o, b}^{b}=\left[\begin{array}{lll}0.1 & -0.2 & 0.3\end{array}\right]^{\top}$ with $\hat{\mathbf{q}}_{o, b}(0)=\left[\begin{array}{llll}1 & 0 & 0 & 0\end{array}\right]^{\top}$ and $\hat{\boldsymbol{\omega}}_{o, b}^{b}=\left[\begin{array}{lll}0 & 0 & 0\end{array}\right]^{\top}$, while Table II shows the different parameters used for the simulation. Let the desired quaternion be given as $\mathbf{q}_{o, d}=\left[\begin{array}{llll}1 & 0 & 0 & 0\end{array}\right]^{\top}$, while the desired angular velocity and acceleration are set to zero. The simulation uses Runge Kutta with fixed step of 0.01 seconds. The Sun sensor is assumed to have a noise of 1.0 degrees, the magnetometer 5.0
TABLE II

PARAMETERS AND VALUES USED FOR THE SIMULATION.

\begin{tabular}{l|ll} 
Parameter & Value & Unit \\
\hline$\mu_{f}$ & $7.9 \cdot 10^{15}$ & $\mathrm{~Wb} \cdot \mathrm{m}$ \\
$G$ & $6.67408 \cdot 10^{-11}$ & $\mathrm{~m}^{3} \mathrm{~kg}^{-1} \mathrm{~s}^{-2}$ \\
$M_{E \text { Earth }}$ & $5.9742 \cdot 10^{24}$ & $\mathrm{~kg}$ \\
$\Delta T$ & 0.01 & $\mathrm{~s}$ \\
$r_{a}$ & $R_{e}+1200$ & $\mathrm{~km}$ \\
$r_{p}$ & $R_{e}+800$ & $\mathrm{~km}$ \\
$R_{e}$ & 6378 & $\mathrm{~km}$ \\
$i$ & 75 & degrees \\
$\Omega$ & 0 & degrees \\
$\omega$ & 0 & degrees \\
$f_{\max }$ & 0.1 & $\mathrm{~N}$ \\
$\beta$ & 1.5 & \\
$\zeta$ & 1 & \\
$k_{p}$ & 2 & \\
$k_{d}$ & 2 & \\
\hline
\end{tabular}

degrees, and the accelerometer 1.0 degree error. The sensors are modeled by converting the quaternion to Euler angles, then add Gaussian noise, and then convert it back to a quaternion. The noisy quaternion then rotates the model vectors $\mathbf{m}^{o}, \mathbf{s}^{o}$ and $\mathbf{a}^{o}$ to the body frame, which then represents the sensor measurements. The sensors are sampled every 0.1 seconds.

Now consider a satellite moving in an elliptical orbit. The objective is to point a sensor mounted along the $\mathbf{x}^{b}$ axis in the nadir direction. This can be achieved by making $\mathbf{q}_{o, b} \rightarrow$ $\left[\begin{array}{llll}1 & 0 & 0 & 0\end{array}\right]^{\top}$, while the angular velocity must go to zero. Figure 2 shows the quaternion and angular velocity of the satellite during the maneuver. It is evident that after about 50 seconds, the objective is achieved. Since the attitude and angular velocity is not measured directly, the Madgwick filter is used to estimate these vectors. Figure 3 shows the estimation error, where both the quaternion error and angular velocity error converge to a bounded set close to zero.

The desired torque calculated using the PD+ controller is mapped to desired thrust values. The thruster firings are shown in Figure 4, where it is observed that initially, some of the thrusters are kept constant on, while others are off. After converging to the desired quaternion and angular velocity, the thrusters are activated almost continuously to maintain the attitude. These activations can be removed by introducing thruster logic such that as long as the attitude error is kept below a threshold the thrusters are not activated.

\section{CONCLUSION}

This paper has shown how to design a complete attitude determination and control system comprising of orbital mechanics, Sun vector model, magnetic field model, actuator dynamics, a PD+ control law, nonlinear attitude dynamics and a Madgwick filter for attitude estimation. Simulations shows very good results, and this can serve as a basis for developing new results for ADCS systems.

\section{ACKNOWLEDGEMENT}

The author would like to thank the reviewers for the good feedback and recommendations that helped improve this paper. 

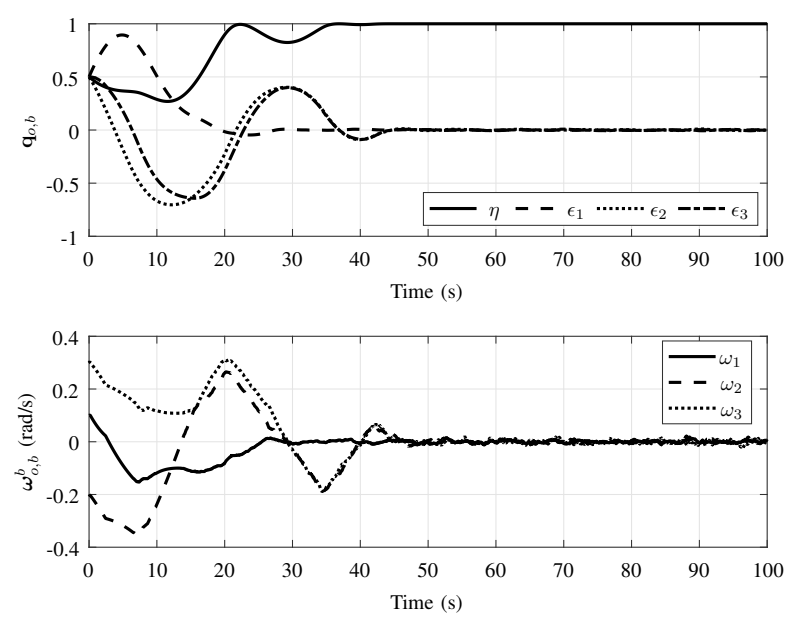

Fig. 2. Attitude and angular velocity during the maneuver.
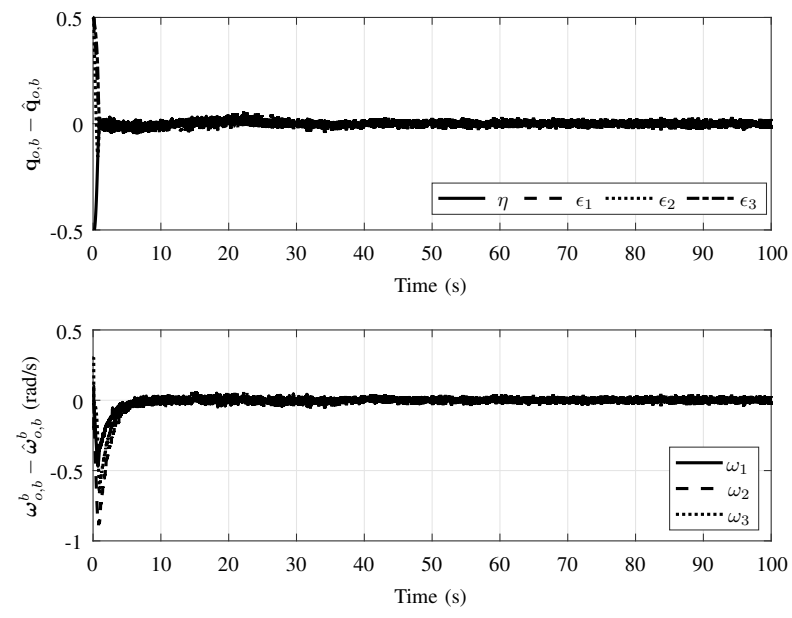

Fig. 3. Estimation error.

\section{REFERENCES}

[1] M. J. Sidi, Spacecraft Dynamics \& Control. Cambridge University Press, 1997.

[2] J. Wertz, ed., Spacecraft Attitude Determination and Control. D. Reidel Publishing Company, 1978.

[3] D. A. Vallado, Fundamentals of Astrodynamics and Applications. Microcosm Press/Springer, 3rd ed., 2007.

[4] F. L. Markley and J. L. Crassidis, Fundamentals of Spacecraft Attitude Determination and Control. Microcosm Press and Springer, 2014.

[5] J. Alarcon, N. Örger, S. Kim, L. Soon, and M. Cho, "Aoba VELOX-IV Attitude and Orbit Control System Design for a LEO Mission Applicable to a Future Lunar Mission," in Proceedings of the 67th International Astronautical Congress, (Guadalajara city, Mexico), 2016.

[6] R. Dechao, S. Tao, C. Lu, C. Xiaoqian, and Z. Yong, "Attitude control system design and on-orbit performance analysis of nano-satellite - Tian Tuo 1," Chinese Journal of Aeronautics, vol. 27, no. 3, pp. 593-601, 2014.

[7] Y. Nakajima, N. Murakami, T. Ohtani, Y. Nakamura, K. Hirako, and K. Inoue, "SDS-4 attitude control system: In-flight results of three axis attitude control for small satellites," IFAC Proceedings Volumes, vol. 16, no. 19 , pp. 283-288, 2013.

[8] M. Fritz, J. Shoer, L. Singh, T. Henderson, J. McGee, R. Rose, and C. Ruf, "Attitude determination and control system design for the CYGNSS microsatellite," in Proceedings of the IEEE Aerospace Conference, (Big Sky, Montana), 2015.

[9] E. Oland, T. Houge, and F. Vedal, "Norwegian student satellite program - HiNCube," in Proceedings of the 22nd Annual AIAA/USU Conference on Small Satellites, (Utah, USA), 2008.
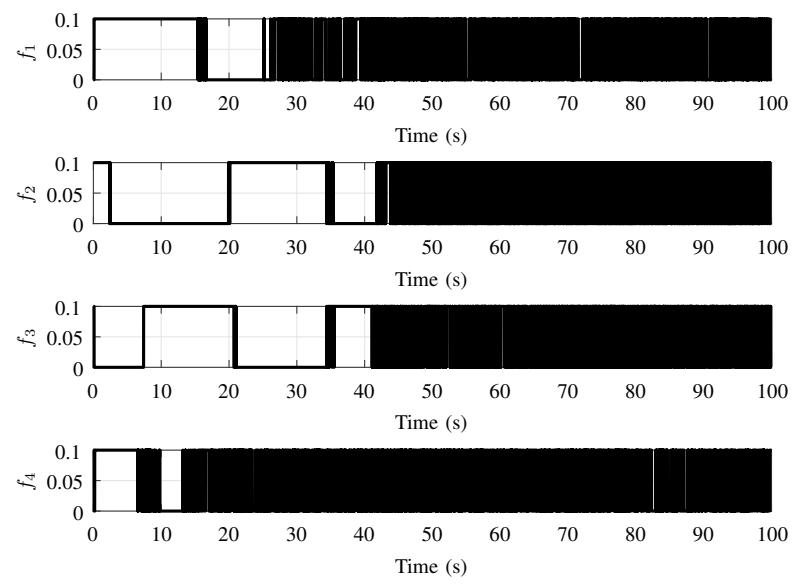

Fig. 4. Thruster firings to control the attitude.

[10] E. Oland and R. Schlanbusch, "Reaction wheel design for cubesats," in Proceedings of the 4th International Conference on Recent Advances in Space Technologies (RAST), (Istanbul, Turkey), 2009.

[11] R. Schlanbusch, E. Oland, and P. J. Nicklasson, "Modeling and simulation of a cubesat using nonlinear control in an elliptic orbit," in Proceedings of the 4th International Conference on Recent Advances in Space Technologies (RAST), (Istanbul, Turkey), 2009.

[12] E. Oland, "A command-filtered backstepping approach to autonomous inspections using a quadrotor," in Proceedings of the 24th Mediterranean Conference on Control and Automation, (Athens, Greece), 2016.

[13] O. Egeland and J. T. Gravdahl, Modeling and simulation for automatic control. Marine Cybernetics, Trondheim, Norway, ISBN 82-92356-01-0, 2002.

[14] W. R. Hamilton, "On quaternions: or a new system of imaginaries in algebra," Philosophical Magazine, vol. 25, pp. 489-495, 1844.

[15] H. Schaub and J. L. Junkins, Analytical Mechanics of Space Systems. American Institute of Aeronautics, 2003.

[16] E. Oland, T. S. Andersen, and R. Kristiansen, "Subsumption architecture applied to flight control using composite rotations," Automatica, vol. 69, pp. 195-200, 2016.

[17] R. Kristiansen, Dynamic synchronization of spacecraft. $\mathrm{PhD}$ thesis, Norwegian University of Science and Technology, Trondheim, Norway, 2008.

[18] E. Thébault et al., "International geomagnetic reference field: the 12th generation," Earth, Planets and Space, vol. 67, no. 79, 2015.

[19] M. Psiaki, "Magnetic torquer atitude control via asymptotic periodic linear quadratic regulation," Journal of Guidance, Control, and Dynamics, vol. 24, no. 2, pp. 386-394, 2001.

[20] B. Paden and R. Panja, "Globally asymptotically stable 'PD+' controller for robot manipulators," International Journal of Control, vol. 47, no. 6, pp. 1697-1712, 1988.

[21] R. Kristiansen, P. J. Nicklasson, and J. T. Gravdahl, "Spacecraft coordination control in 6DOF: Integrator backstepping vs. passivity-based control," Automatica, vol. 44, no. 11, pp. 2896-2901, 2008.

[22] W. Hahn, Stability of Motion. Springer-Verlag Berlin Heidelberg New York, ISBN: 978-3-642-50087-9, 1967.

[23] S. Madgwick, A. Harrison, and R. Vaidyanathan, "Estimation of IMU and MARG orientation using a gradient descent algorithm," in Proceedings of the 2011 IEEE International Conference on Rehabilitation Robotics, (Zurich, Switzerland), 2011.

[24] M. D. Shuster and S. D. Oh, "Three-axis attitude determination from vector observations," Journal of Guidance and Control, vol. 4, no. 1, pp. 70-77, 1981.

[25] J. L. Farrell, "Attitude determination by kalman filtering," Automatica, vol. 6, no. 3, pp. 419-430, 1970.

[26] R. Mahony, T. Hamel, and J. Pflimlin, "Nonlinear complementary filters on the special orthogonal group," IEEE Transactions on Automatic Control, vol. 54, no. 5, pp. 1203-1217, 2008. 